SHORT REPORT

\title{
Meningeal derived cerebrospinal fluid proteins in different forms of dementia: is a meningopathy involved in normal pressure hydrocephalus?
}

\author{
J Brettschneider, M W Riepe, H-F Petereit, A C Ludolph, H Tumani
}

J Neurol Neurosurg Psychiatry 2004;75:1614-1616. doi: 10.1136/jnnp.2003.026013

\begin{abstract}
Objectives: In animal models and in vitro studies leptomeninges have been shown to be the origin of neurotrophic substances that support the survival and growth of neuronal cells. Because dementia is associated with neuronal loss, we investigated whether leptomeningeal dysfunction may be involved in the pathogenesis of dementia disorders.

Methods: We analysed the cerebrospinal fluid (CSF) concentrations of the leptomeningeal derived $\beta$ trace protein, $\beta 2$ microglobulin, and cystatin C.

Results: There was a statistically significant difference of the CSF $\beta$ trace protein levels among different groups. Patients with idiopathic normal pressure hydrocephalus (NPH) (17.5 (SD 4.3) $\mathrm{mg} / \mathrm{l}$ ) showed significantly lower CSF $\beta$ trace protein levels than patients with Alzheimer's disease (23.8 (6.2) $\mathrm{mg} / \mathrm{l})$, depression (24.2 (7.3) $\mathrm{mg} / \mathrm{l})$, and normal controls (25.3 (4.9) mg/l). To patients with vascular dementia $(20.1$ (5.6) $\mathrm{mg} / \mathrm{l})$ and frontotemporal dementia (21.9 (7.0) $\mathrm{mg} / \mathrm{l})$, the difference was not significant. There was no significant difference regarding the CSF and serum concentrations of $\beta 2$ microglobulin or cystatin $C$ among the different groups.

Conclusions: We conclude that leptomeningeal dysfunction may be involved in certain types of dementia such as NPH and that reduced CSF $\beta$ trace protein levels in patients with $\mathrm{NPH}$ may aid in differentiating this difficult to diagnose disorder from other syndromes such as Alzheimer's disease.
\end{abstract}

$\mathrm{T}$ he leptomeninges have been shown to support the survival and growth of neuronal cells and to produce neurotrophic factors. ${ }^{1-4}$ Because dementia is associated with neuronal loss, leptomeningeal dysfunction may be a possible pathomechanism in dementia syndromes. In Alzheimer's disease, the leptomeninges have been shown to be a major site of $\beta$ amyloid deposition. ${ }^{5}$ In idiopathic normal pressure hydocepalus (NPH), several studies showed a leptomeningeal fibrosis of unknown origin. ${ }^{6}$ To assess meningeal function in vivo, we measured cerebrospinal fluid (CSF) proteins produced by the leptomeninges. ${ }^{3}$

$\beta$ Trace protein has been shown to be mainly synthesised in the spinal leptomeninges and pachymeninx. ${ }^{89}$ It seems to have a function related to prostaglandin metabolism ${ }^{10}$ and to act as a carrier protein for retinoids. ${ }^{11} \beta 2$ microglobulin is a cell membrane associated peptide expressed on the surface of most nucleated cells that may be involved in immunological reactions. ${ }^{12}$ Cystatin $\mathrm{C}$ is an important extracellular inhibitor of cysteine proteinases. ${ }^{13}$

The aim of this study was to investigate concentrations of CSF proteins with a known leptomeningeal origin in various subtypes of dementia to determine whether signs of meningeal dysfunction are present.

\section{METHODS}

Paired lumbar CSF and serum samples were obtained from 96 patients from the department of neurology, University Hospital of Ulm, with different subtypes of dementia.

We examined 30 patients ( 18 female, 12 male) fulfilling the diagnostic criteria of Alzheimer's disease as described by McKhann et al. ${ }^{14}$ Furthermore, we investigated 13 patients (6 female, 7 male) with vascular dementia, 6 patients ( 3 female, 3 male) with frontotemporal dementia (FTD), 13 patients ( 6 female, 7 male) with pseudo-dementia caused by depression, and 19 patients ( 11 female, 8 male) with idiopathic forms of $\mathrm{NPH}$. The diagnosis of the non-Alzheimer's disease groups of dementia was based on the Diagnostic and statistical manual of mental disorders, fourth edition (DSM-IV). ${ }^{15}$ The diagnosis of $\mathrm{NPH}$ was based on the clinical triad of gait disturbance, dementia, and urinary incontinence or increased urinary urgency. ${ }^{16}$ Patients were required to show typical criteria in neuroimaging. ${ }^{16}$ Clinical improvement after diagnostic lumbar CSF puncture was taken as a confirmation of the diagnosis to provide greater diagnostic security, although this implied that some cases of NPH with a negative tap test may have been excluded.

CSF and serum samples were obtained from 15 (8 female, 7 male) individuals approximately age matched to patients with Alzheimer's disease, who were normal controls. On clinical examination, neuroimaging (MRI (magnetic resonance imaging) or CT (computed tomography)), and CSF analysis they did not show any signs of a pathological process affecting the central nervous system.

Informed consent of all patients and the approval of the ethical committee of the University of Ulm were obtained. All CSF and serum samples were analysed in our CSF laboratory. $\beta$ trace protein, $\beta 2$ microglobulin, and cystatin $\mathrm{C}$ were measured using the test kit (immunonephelometric assay using polyclonal rabbit IgG antibodies) of Behringwerke AG (Marburg, Germany). Details on the assay procedure and validity data have been described previously by our group. ${ }^{18}$

Statistical analysis was carried out using a one way analysis of variance (ANOVA), followed by an all pair wise multiple comparison procedure (Tukey test) in case of a statistically significant difference. As a normality test, the Kolmogorov-Smirnov test was applied. In case there was no normal distribution, a Kruskal-Wallis ANOVA was applied. Values $<0.05$ are referred to as statistically significant.

Abbreviations: CSF, cerebrospinal fluid; FTD, frontotemporal dementia; $\mathrm{NPH}$, normal pressure hydrocephalus 
Table 1 Meningeal markers in cerebrospinal fluid of patients with different subtypes of dementia

\begin{tabular}{|c|c|c|c|c|c|c|c|c|}
\hline & \multirow[b]{2}{*}{$\mathbf{n}$} & \multirow{2}{*}{$\frac{\text { Age }}{\text { Mean (SD) }}$} & \multicolumn{2}{|c|}{$\beta$ Trace protein $(\mathrm{mg} / \mathrm{l})$} & \multicolumn{2}{|c|}{ B2 Microglobulin (mg/l) } & \multicolumn{2}{|c|}{ Cystatin C (mg/l) } \\
\hline & & & Mean (SD) & Range & Mean (SD) & Range & Mean (SD) & Range \\
\hline Alzheimer's disease & 30 & $69.8(8.7)$ & $23.8(6.2)$ & $13.1-35.3$ & $1.3(0.3)$ & $0.8-1.9$ & $3.5(1.2)$ & $1.4-6.4$ \\
\hline Vascular dementia & 13 & $75.4(4.7)$ & $20.1(5.6)$ & $13.8-30.0$ & $1.4(0.3)$ & $0.9-1.8$ & $3.0(1.3)$ & $1.0-4.8$ \\
\hline Frontotemporal dementia & 6 & $60.8(7.8)$ & $21.9(7.0)$ & $11.2-29.2$ & $1.3(0.3)$ & $0.9-1.6$ & $3.3(1.7)$ & $1.5-5.9$ \\
\hline Depression & 13 & $61.5(9.1)$ & $24.2(7.3)$ & $17.5-44.3$ & $1.3(0.6)$ & $0.9-2.9$ & $3.3(1.6)$ & $1.4-5.5$ \\
\hline $\begin{array}{l}\text { Normal pressure } \\
\text { hydrocephalus }\end{array}$ & 19 & $76.6(6.7)$ & $17.5(4.3)$ & $10.3-24.5$ & $1.3(0.5)$ & $0.2-2.1$ & $3.1(1.0)$ & $1.1-4.9$ \\
\hline Controls & 15 & $73.7(6.4)$ & $25.3(4.9)$ & $14.8-36.2$ & $1.5(0.3)$ & $0.8-2.0$ & $4.3(1.3)$ & $2.5-6.5$ \\
\hline
\end{tabular}

\section{RESULTS}

In routine CSF analysis all patients and controls revealed normal results for cell count, $\mathrm{CSF} /$ serum concentration ratio of albumin $\left(\mathrm{Q}_{\mathrm{ALB}}\right)$ representing blood-CSF barrier function, and oligoclonal IgG bands. Levels of $\beta$ trace protein, $\beta 2$ microglobulin, and cystatin $\mathrm{C}$ in CSF showed a positive correlation with age and $\mathrm{Q}_{\mathrm{ALB}}$ in controls and patients.

There was a statistically significant difference of the CSF $\beta$ trace protein levels among the different groups $(p=0.001$, ANOVA). Patients with NPH showed significantly lower $\beta$ trace protein levels than normal controls $(p=0.003$, Tukey test), patients with Alzheimer's disease $(p=0.005$, Tukey test), and patients with pseudo-dementia caused by depression ( $p=0.021$, Tukey test) (table 1 , fig 1$)$. They were lower than in patients with vascular dementia $(\mathrm{p}=0.817$, Tukey test) or FTD $(p=0.582$, Tukey test $)$, although these differences were not statistically significant. Among the non-NPH patients, no significant difference could be found. Likewise, there was no significant difference regarding $\beta$ trace protein serum levels $(p=0.53$, Kruskal-Wallis test, data not shown).

Neither CSF ( $\mathrm{p}=0.687$, ANOVA) $(\mathrm{p}=0.228$, ANOVA) nor serum levels $(p=0.538$, ANOVA) $(p=0.083$, ANOVA) of $\beta 2$ microglobulin and cystatin $C$ showed a significant difference among the different groups (see table 1).

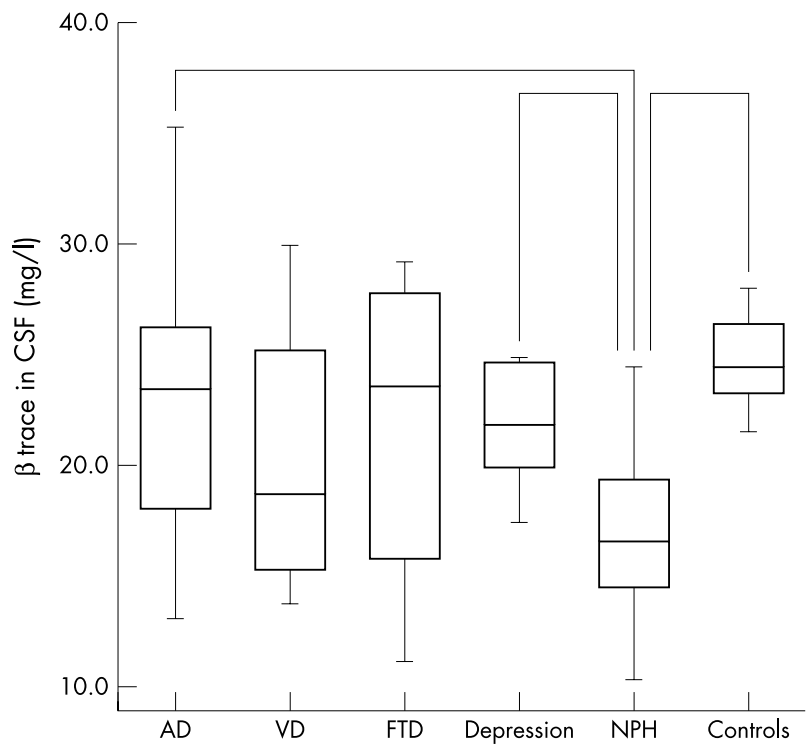

Figure 1 Cerebrospinal fluid $\beta$ trace protein concentrations in patients with different causes of dementia and normal controls, brackets indicate statistical significant difference $(p<0.05)$. AD, Alzheimer's disease; VD, vascular dementia; FTD, frontotemporal dementia; NPH, normal pressure hydrocephalus.

\section{DISCUSSION}

The CSF levels of the meningeal markers were not found to be different between patients with Alzheimer disease, vascular dementia, FTD, and normal controls. In contrast, patients with NPH showed considerably lower $\beta$ trace protein concentrations. As an explanation, a dilution effect due to the enlargement of the ventricular spaces in NPH does not seem plausible as the other meningeal markers were not affected similarly.

The leptomeninges are thought to be a main origin of $\beta$ trace protein. ${ }^{8}{ }^{18}$ Reduced CSF $\beta$ trace protein levels could therefore mirror a pathological process affecting the meninges in NPH. As not all meningeal markers in our study were affected to the same extent, the function of the meninges may only be partially impaired. Furthermore, of the three investigated markers, only $\beta$ trace protein seems to originate mainly from the meninges, ${ }^{3} 818$ while cystatin $C$ and $\beta 2$ microglobulin are released from non-meningeal structures as well. Their CSF concentration may therefore be less vulnerable to meningeal dysfunction than $\beta$ trace protein.

The reason for a possible meningeal dysfunction in NPH remains unclear. Leptomeningeal biopsies have failed to show $^{19} 20$ an unequivocal association of leptomeningeal fibrosis with a disturbed CSF flow. ${ }^{16}$ A meningeal dysfunction as indicated by decreased CSF $\beta$ trace protein levels may be involved in the pathogenesis of idiopathic NPH by contributing to a greater resistance to CSF outflow. However, this hypothesis requires further proof. Nevertheless, our data are in line with the notion that several types of dementia, including NPH and Alzheimer's disease, may be parts of a continuum representing CSF circulatory dysfunction as hypothesised recently. ${ }^{21}$

As the CSF level of $\beta$ trace protein was significantly lower in NPH than in Alzheimer's disease and asymptomatic people, it might be useful to also test for a differentiation between NPH and Alzheimer's disease. However, the test could not differentiate patients with NPH from those with vascular dementia, as concentrations of $\beta$ trace protein in both groups were not significantly different.

Because the important issue of NPH diagnosis is predicting shunt responsiveness, correlation of $\beta$ trace protein levels with responsiveness after a shunt are warranted in future studies.

\section{ACKNOWLEDGEMENTS}

JB and HT have equally contributed to the design, analysis, data interpretation of this study, and writing the article. MWR and HFP performed clinical investigations and were involved in discussions of the results. ACL was involved in discussions of the results. We thank Jan Vanneste and the unknown reviewers for their helpful comments. This work was supported in part by local university funding for HT.

This paper is dedicated to the pioneer of clinical CSF research Professor Klaus Felgenhauer, who died on 8 July 2002. 


\section{Authors' affiliations}

J Brettschneider, M W Riepe, A C Ludolph, H Tumani, Department of Neurology, University of Ulm, Ulm, Germany

H-F Petereit, Department of Neurology, University of Köln, Köln, Germany

Conflict of interest: none declared

Correspondence to: $\mathrm{Dr} \mathrm{H}$ Tumani, Klinik für Neurologie der Universitä Ulm, Oberer Eselsberg 45, 89091 Ulm, Germany; hayrettin.tumani@ medizin.uni-ulm.de

Received 15 August 2003

In revised form 15 January 2004

Accepted 16 January 2004

\section{REFERENCES}

1 Ueda S, Tanabe T, Ihara N, et al. Immunohistochemical study of fetal raphe samples transplanted into the leptomeningeal tissues of 5,6-dihydroxytryptamine-treated adult rats. Cell tissue res 1989;256:457-63.

2 Ishikawa K, Kabeya K, Shinoda M, et al. Meninges play a neurotrophic role in the regeneration of vasopressin nerves after hypophysectomiy. Brain Res 1995:677:20-8.

3 Ohe Y, Ishikawa K, Itoh Z, et al. Cultured leptomeningeal cells secrete cerebrospinal fluid proteins. J Neurochem 1996;67:964-71

4 Canalis E, McCarthy T, Centrella M. A bone derived growth factor isolated from rat calvariae is beta2 microglobulin. Endocrinology 1987; 121:1198-200.

5 Wisniewski HM, Wegiel J. $\beta$-Amyloid formation by myocytes of leptomeningeal vessels. Acta Neuropathol Berl 1994;87:233-41.

6 Bech RA, Juhler M, Waldemar G, et al. Frontal brain and leptomeningea biopsy specisms correlated with cerebrospinal fluid outflow resistance and Beta-trace-wave activity in patients suspected of normal pressure hydrocephalus. Neurosurg 1997;40:497-502

7 Di Rocco C, Di Trapani G, Maira G, et al. Anatomo-clinical correlations in normotensive hydrocephalus. Reports on three cases. J Neurol Sci 1977:33:437-52.
8 Urade $Y$, Katahama K, Ohishi J, et al. Dominant expression of m-RNA for prostaglandin D-synthetase in leptomeninges, choroid plexus and oligodendrocytes of the adult rat brain. Proc Natl Acad Sci 1993;90:9070-4.

9 Blödorn B, Brück W, Tumani $\mathrm{H}$, et al. Expression of the beta-trace protein in human pachymeninx revealed by in situ hybridisation and immunocytochemistry. J Neurosci Res 1999;57:730-4.

10 Watanabe K, Urade Y, Mäder M, et al. Identification of beta-trace as prostaglandin D synthase. Biochem Biophys Res Commun 1994:203:1110-6.

11 Tanaka T, Urade Y, Kimura H, et al. Lipocalin-type prostaglandin D sythase (beta-trace) is a newly recognized type of retinoid transporter. J Biol Chem 1997;25:15789-95.

12 Starmans JJ, Vos J, van der Helm HJ. The beta 2-microglobulin content of the cerebrospinal fluid in neurological disease. J Neurol Sci 1977;33:45-9

13 Abrahamson M, Buttle DJ, Mason RW, et al. Regulation of cystatin C activity by serine proteases. Biomed Biochem Acta 1991;50:587-93.

14 McKhann G, Drachman D, Folstein M et al. Clinical diagnosis of Alzheimer's disease: report of the NINCDS-ADRDA Work Group under the auspices of Health and Human Services Task Force on Alzheimer's Disease. Neurology 1984;34:939-44.

15 American Psychiatric Association. Diagnostic and Statistical Manual of Mental Disorders - Fourth Edition (DSM-IV). Washington DC: American Psychiatric Association, 1994.

16 Vanneste JA. Diagnosis and management of normal pressure hydrocephalus. J Neurol 2000:247:5-14

17 Bradley WG Jr. Diagnostic tools in hydrocephalus. Neurosurg Clin N Am 2001;12:661-84.

18 Tumani H, Nau R, Felgenhaver K. Beta-trace protein in cerebrospinal fluid: a blood-CSF barrier-related evaluation in neurological diseases. Ann Neurol 1998:44:82-9

19 DeLand F, James AE, Ladd DJ, et al. Normal pressure hydrocephalus: a histologic study. Am J Clin Pathol 1972;58:58-63.

20 Bech RA, Waldemar G, Gjerris F, et al. Shunting effects in patients with idiopathic normal pressure hydrocephalus; correlation with cerebral and leptomeningeal biopsy findings. Acta Neurochir 1999;141:633-9.

21 Silverberg GD, Mayo M, Saul T, et al. Alzheimer's disease, normal-pressure hydrocephalus, and senescent changes in CSF circulatory physiology: a hypothesis. Lancet Neurol 2003;2:506-11. 